\title{
Scalar Source in Circular Motion Interacting with Massive Klein-Gordon Field in Minkowski Spacetime
}

\author{
Luís C. B. Crispino and Damião P. Meira Filho \\ Departamento de Física, Universidade Federal do Pará, 66075-110, Belém, PA, Brazil
}

(Received on 15 October, 2005)

\begin{abstract}
We analyze the scalar radiation emitted by a source in uniform circular motion in Minkowski spacetime interacting with a massive Klein-Gordon field. We assume the source rotating around a central object due to a Newtonian force. By considering the canonical quantization of this field, we use perturbation theory to compute the radiation emitted at the tree level. Regarding the initial state of the field as being the Minkowski vacuum, we compute the emission amplitude for the rotating source, assuming it as being minimally coupled to the massive Klein-Gordon field. We then compute the power emitted by the swirling source as a function of its angular velocity, as measured by asymptotic static observers.
\end{abstract}

In a previous work one of the authors together with Higuchi and Matsas have analyzed the radiation emitted by a source rotating around a massive object and interacting with a massless scalar field [1]. More recently this analysis has been extended to the electromagnetic case [2]. In the present work we investigate how the mass of the scalar field influences the emission rate and the power emitted by a swirling source rotating around a massive object, due to a Newtonian gravitational force. We assume natural units $c=G=1$ and metric signature $(+---)$.

We start by exhibiting the classical solutions to the massive Klein-Gordon field equation in Minkowski spacetime, $\left(\nabla^{\mu} \nabla_{\mu}+m^{2}\right) u_{\omega l p}=0$, in spherical polar coordinates

$$
\begin{aligned}
& u_{\omega l p}\left(x^{\mu}\right)=\left(\frac{\sqrt{\omega^{2}-m^{2}}}{\pi}\right)^{\frac{1}{2}} \times \\
& j_{l}\left(r \sqrt{\omega^{2}-m^{2}}\right) Y_{l p}(\theta, \varphi) \exp (-i \omega t) \quad(\omega \geq m),
\end{aligned}
$$

with positive frequency $\omega$, and angular momentum quantum numbers $l$ and $p$. Here $j_{l}(r)$ are spherical modified Bessel functions and $Y_{l p}(\theta, \varphi)$ are the scalar spherical harmonics. These positive-frequency orthogonal modes have been normalized through Klein-Gordon inner product

$$
\sigma_{K G}(\phi, \psi)=i \int_{\Sigma^{(3)}} d \Sigma^{(3)} n^{\mu}\left[\phi^{*}\left(\nabla_{\mu} \psi\right)-\left(\nabla_{\mu} \phi^{*}\right) \psi\right]
$$

such that $\sigma_{K G}\left(u_{\omega l p}, u_{\omega^{\prime} l^{\prime} p^{\prime}}\right)=\delta_{l l^{\prime}} \delta_{p p^{\prime}} \delta\left(\omega-\omega^{\prime}\right)$. The quantum scalar field is given by the usual Fourier expansion of the positive- and negative-frequency modes

$$
\widehat{\Phi}\left(x^{\mu}\right)=\sum_{l=0}^{\infty} \sum_{p=-l}^{l} \int_{m}^{\infty} d \omega\left[u_{\omega l p}\left(x^{\mu}\right) \hat{a}_{\omega l p}+u_{\omega l p}^{*}\left(x^{\mu}\right) \hat{a}_{\omega l p}^{\dagger}\right]
$$

so that the non-vanishing commutation relations between the creation and annihilation operators are

$$
\left[\hat{a}_{\omega l p}, \hat{a}_{\omega^{\prime} l^{\prime} p^{\prime}}\right]=\delta_{l l^{\prime}} \delta_{p p^{\prime}} \delta\left(\omega-\omega^{\prime}\right) .
$$

The quantum field states are obtained by applying the creation operators to the Minkowski vacuum $|0\rangle$, defined by $\hat{a}_{\omega l p}|0\rangle=0$. Our rotating source, represented by $J\left(x^{\mu}\right)=$

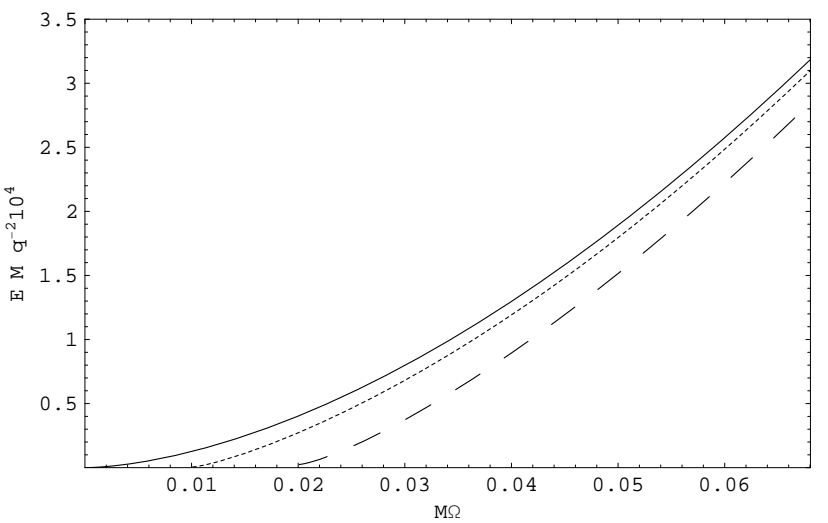

FIG. 1: The contribution to the emission rate $E$ coming from particles with angular momentum up to $l=3$ is plotted as a function of angular velocity $\Omega$ of the rotating source, as measured by asymptotic static observers, for the cases with $m=0$ (continuous line), $m M=0.01$ (dotted line) and $m M=0.02$ (dashed line). $M \Omega$ ranges from 0 up to 0.068 (associated with the circular orbit at $R=6 M$ ).

$\frac{q}{R^{2} \gamma} \delta(r-R) \delta\left(\theta-\frac{\pi}{2}\right) \delta(\varphi-\Omega t)$, is regarded as minimally coupled to the field $\widehat{\Phi}\left(x^{\mu}\right)$, through the interaction action $\widehat{S}_{I}=$ $\int d^{4} x \sqrt{-g} J \widehat{\Phi}$. Here $\gamma=1 / \sqrt{\left(1-R^{2} \Omega^{2}\right)}$. Keplerian orbits of the source at a fixed radius $R$, with angular velocity $\Omega$ as measured by asymptotic static observers, are characterized by $R=\left(M / \Omega^{2}\right)^{1 / 3}$, where $M$ is the mass of the central object, which attracts the source through a Newtonian gravitational force. The total emission rate of one quantum of the field into the vacuum is given by

$$
\begin{aligned}
E=\sum_{l=1}^{\infty} \sum_{p=1}^{l} \int_{m}^{\infty} d \omega \frac{\left|\left\langle 1 ; \omega l p\left|i \widehat{S}_{I}\right| 0\right\rangle\right|^{2}}{T} \\
=\sum_{l=1}^{\infty} \sum_{p=1}^{l} \frac{2 q^{2} \sqrt{p^{2} \Omega^{2}-m^{2}}}{\gamma^{2}} \times \\
\left|j_{l}\left(\left(\frac{M}{\Omega^{2}}\right)^{\frac{1}{3}} \sqrt{(p \Omega)^{2}-m^{2}}\right)\right|^{2}\left|Y_{l p}\left(\frac{\pi}{2}, \Omega t\right)\right|^{2},
\end{aligned}
$$

where $T \equiv 2 \pi \delta(0)=\int_{-\infty}^{\infty} d t$ is the total time measured by the asymptotic static observers. In Fig. 1 we plot the emission rate for two different values of the product $m M$, as well as 


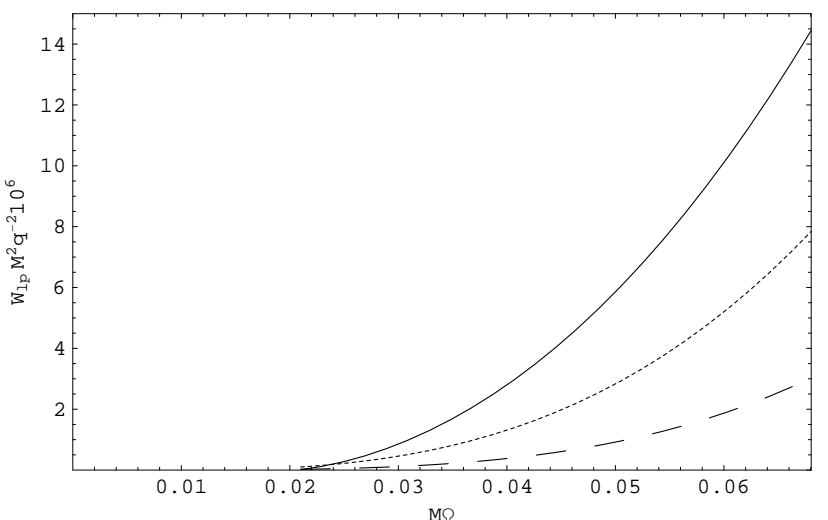

FIG. 2: $W_{l p}$ is plotted as a function of $\Omega$ for different choices of $l$ and $p$, with $m M=0.02$. The contribution from $l=p=1$ is given by the continuous line, the one from $l=p=2$ by the dotted line, whereas the contribution $l=p=3$ is given by the dashed line. The main contribution to the emitted power comes from modes with angular momentum $l=p=1$. The larger $l$ is, the smaller is the contribution to the total radiated power.

for $m=0$. We see that for a fixed value of $\Omega$, the emission rate is smaller in the massive case than in the massless case. Moreover, the greater the value of $m M$, the less is the emission rate for a fixed angular velocity of the source.

The next step is to compute the power emitted $W$ by the swirling source. For a fixed value of the angular momentum quantum numbers $l$ and $p$ we have that

$$
\begin{aligned}
& W_{l p}=\int_{m}^{\infty} d \omega \omega \frac{\left|\left\langle 1 ; \omega l p\left|i \widehat{S}_{I}\right| 0\right\rangle\right|^{2}}{T} \\
= & \frac{2 q^{2} p \Omega \sqrt{p^{2} \Omega^{2}-m^{2}}}{\gamma^{2}} \times \\
& \left|j_{l}\left(\left(\frac{M}{\Omega^{2}}\right)^{\frac{1}{3}} \sqrt{(p \Omega)^{2}-m^{2}}\right)\right|^{2}\left|Y_{l p}\left(\frac{\pi}{2}, \Omega t\right)\right|^{2} .
\end{aligned}
$$

In Fig. 2 we plot $W_{l p}$ as a function of the angular velocity of the source, for increasing values of $l$. We see that the major contribution is given by the mode with $l=p=1$. For a fixed value of $l$ the dominant contribution comes from $l=p$.
Moreover, modes with odd values of $l+p$ have vanishing contributions to the emitted power. Modes with $l=p=0$ are not emitted by the rotating source. The total emitted power is obtained by adding up all the contributions from all possible values of $l$ and $p$, namely $W=\sum_{l=1}^{\infty} \sum_{p=1}^{l} W_{l p}$. In Fig. 3 we plot the total power emitted by the rotating source for $m=0$ and also for two non-vanishing values of the product $m M$. Analogously to what happens for the emission rate, for a fixed value of $\Omega$, the emitted power is also smaller for larger values of $m M$.

The case of a source, minimally coupled to massive scalar field, swirling around a Schwarzschild black hole is presently under investigation.

The authors are grateful to G. E. A. Matsas and J. Cantiñeiras for profitable discussions; and to Conselho Nacional de Desenvolvimento Científico e Tecnológico (CNPq)

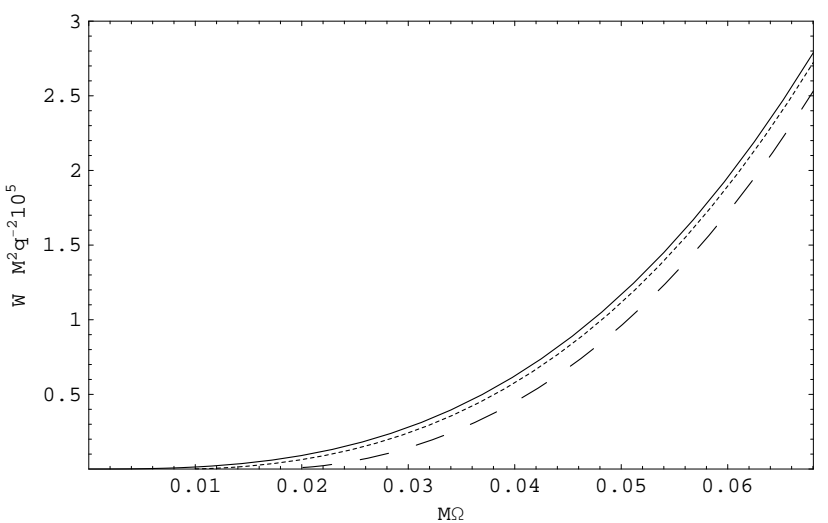

FIG. 3: The total power $W$ emitted by the source rotating around the massive object due to a Newtonian force is plotted as a function of the angular velocity $\Omega$. The $l$ summation in the definition of $W$ is performed up to $l=3$. One of the plots have been performed for the massless scalar field ( $m=0$, continuous line), while for the other two we have chosen $m M=0.01$ (dotted line) and $m M=0.02$ (dashed line). Again $M \Omega$ ranges from 0 up to 0.068 .

for partial financial support. D. P. M. F. would like to acknowledge also partial financial support from Coordenação de Aperfeiçoamento de Pessoal de Nível Superior (CAPES).
[1] L. C. B. Crispino, A. Higuchi, and G. E. A. Matsas, Class. Quantum Grav. 17, 19 (2000).

[2] J. Castiñeiras, L. C. B. Crispino, R. Murta, and G. E. A. Matsas,
Phys. Rev. D 71, 104013 (2005). 